\title{
A LA SOMBRA DE LA PRODUCCIÓN: APROXIMACIONES Y PUESTA EN VALOR DE LA CATÁBASIS, EXTENSIÓN OCULTA DEL PROCESO CREAT IVO
}

\section{Rakel Gómez Vázquez}

Universidad del País Vasco / Euskal Herriko Unibertsitatea. Dpto. Didáctica de la Expresión Musical, Plástica y Corporal

\section{Resumen}

Este artículo tiene como objetivo principal la puesta en valor y defensa del fracaso, la duda, las contradicciones y las paradojas inherentes a los procesos de producción e investigación artística, y su puesta en escena como método de construcción de saberes. Partiremos en esta reflexión de la presentación del caso de "El club Fénix" como ejemplo de la puesta en práctica, en espacios experimentales y abiertos, de metodologías artísticas que abren espacio a la discusión acerca de los problemas que atraviesa la creación artística en los procesos de producción. A partir de la presentación de este caso se revisarán las problemáticas a las que el arte se enfrenta desde la concepción académica del saber y de la construcción de conocimiento, así también como las consecuencias y posibilidades para la comunicabilidad de este saber.

\section{Palabras-clave: INVESTIGACIÓN ARTÍSTICA; FRACASO; CONOCI- MIENTO; CATÁBASIS; CREACIÓN COMO INVESTIGACIÓN}

\section{IN THE SHADOW OF PRODUCTION: AN APPROXIMATION AND VALUATION OF CATABASIS, A HIDDEN EXTENSION OF THE CREATIVE PROCESS}

\section{Abstract}

This paper aims at highlighting and defending the value of failure, doubt, contradiction and paradox within artistic production and research processes. The article further focuses on showcasing these features as inherent and valid methods for knowledge-building. The report starts with the analysis of "The Phoenix club" as a practical example of an open and experimental space, where artistic methodologies enable further spheres of discussion regarding the problems faced by artistic production processes. Moreover, this analysis leads to a review of the difficulties related to art itself from an academic knowledge and competence-building standpoint, thus delving into the consequences and possibilities for communicating such expertise.

Keywords: ARTIST RESEARCH; FAILURE; KNOWLEDGE; CATABASIS; PRACTICE-BASED RESEARCH

\footnotetext{
Gómez Vázquez, Rakel. "A la sombra de la producción: Aproximaciones

y puesta en valor de la catábasis, extensión oculta del proceso

creativo". AusArt 6 (1): 261-272. D0I: 10.1387/ausart.19464
}

\section{AUSART}




\section{Club Fénix}

Ciclo "Terror en verano"

[A la sombra de la producción] Club Fénix carece del optimismo propio de los proyectos grupales y colabora-
tivos, pero se ha visto en la desganada obligación de despejar la sala de un club
para que artistas de todas las disciplinas lleguen, con el caballo cansado y la
mente embotada, a ofrecer lo peor de su obra. Se trata de replegar esfuerzos hacia
zonas oscuras y negativizadas de la producción con el objetivo de visibilizar (sin
polución exhibicionista, voyeurista o autovictimizante) aquellas zonas áridas, se-
cas o empantanadas, en un entorno respetuoso de trabajo, de baja estridencia y
con concentración. La riqueza de sentido ba caido en el desasssiego de girar sobre si misma.
El futuro es algo higubre y no auspicia nada fértil.
No todo defecto repectido es virtud.
No toda recurrencia muestra la perseverancia de algo relevante. La riqueza de sentido ba caido en el desasssiego de girar sobre si misma.
El futuro es algo higubre y no auspicia nada fértil.
No todo defecto repectido es virtud.
No toda recurrencia muestra la perseverancia de algo relevante. La riqueza de sentido ba caido en el desasssiego de girar sobre si misma.
El futuro es algo higubre y no auspicia nada fértil.
No todo defecto repectido es virtud.
No toda recurrencia muestra la perseverancia de algo relevante. No toda recurrencia muestra la perseverancia de algo relevante.
No todas las apariciones azarosas tienen un motivo que las justifigue. No toda espontaneidad es pertinente. No todas las lecturas aportan algo. No toda compañia es enriquecedora.

Poema chino anónimo del s. XVIII

El entorno estarí severamente reglamentado. Frente a la hipérbole y la estridencia imperantes, hemos decidido regular esta porción de realidad para que imperen la sobriedad, decoro, la austeridad, la moderación, la prudencia.

$\mathrm{El}$ año es largo y lleno de desagradables sorpresas. Las estaciones -como el ritmo de una respiración enorme, caprichos y ajena- dan nombre a los ciclos en los cuales se desplegará actividad de Club Fénix “Terror en verano" "P Pavor en otono", "Temor en invierno", "Pánico en primavera".

Arrastramos hasta ustedes el primer ciclo, una serie de encuentros en los que, durante 3 meses, los participantes podrán arrimarse a comentar miserias propias $y$ ajenas, $y$ escucha palabras ay palabras $y$ más palabras sobre las áreas más bloqueadas, amenazadas, destructivas $y$ paralizantes de la propis producción.

\section{Síntesis de la dinámica}

Duración: 3 meses.

Cantidad total de encuentros por ciclo: 6

Participantes por ciclo: 12 fracasantes y 12 expectantes.

Descifrantes: 1 por encuentro.

Descifrantes: 1 por encuentro.
Frasantes: Participarin del ciclo completo

Expectantes: Podrán inscribirse a los encuentros de form esporídica.

Fechas
Enero 2018: Martes 30 - 19,00 a 22:00 hs.

Febrero 2018: Martes 6 y Martes $20-19.00$ a $22: 00$ hs. Marzo 2018: Martes 6 y Martes $20-19.00$ a $22: 00$ hs. Abril 2018: Martes 3- 19:00 a 22:00 hs.

Comercio

Los honorarios de Club Fénix para los fracasantes será de $\$ 1400$ mensuales.

Los honorarios de Club Fénix para los expectantes serín

Actividad

"Terror en verano" consta de 6 encuentros. En cada uno de ellos, 2 participantes o fracasantes expondrán un problema en una obra, un proyecto (o un aspecto) que no funcione, que se encuentre paralizado, inconsistente, débil en su concepción y/o realización y/o consecución.

Recibirín, por parte de un descifrante una mirada, un indicio hacia una zona de excavación, un martilleo serio en los espacios fosilizados, la pista hacia un emplazamic
des que lo sostienen.

No creemos en muchas cosas, pero tenemos la sospecha de que el abandono del anichamiento de la mirada ayudará a dejar al descubierto (a la intemperic, al desamparo) los temas de la obra. Para eso, y porque entendemos que a la vez que se reproducen los discursos sobre las obras, hay una imposibilidad fundante de comunicar aquello de lo que la obra trata, hemos convocado a personas de las más diversas áreas para que miren, miren, miren, piensen, piensen, piensen y hablen, hablen, hablen. Una devolución siempre en nectivo - asi el espiritu de Club Fénix-- una devolución chenter una dewhe

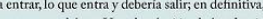

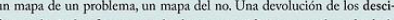
frantes hacia los fracasantes hecha en otro idioma: un relato desde la transdisciplinariedad. Una voz lenta y pausada, ajena, exótica
ca de la obra, que se despliega desde tierras remotas y frescas.

Tendrán acceso (como testigos) a esta deconstrucción y reconstrucción 12 expectantes, que no podrán manifestarse más que en la acción de presenciar, escuchar y, tal vez, realizar anotaciones, Sus limitaciones en la intervención se verín recompensadas por su libertad en el tiempo. Podrán inscribirse a encuentros sueltos, sin una continuidad, esporídicos; muy al uso de los tiempos.

Descifrantes del ciclo "Terror en verano"

Graciela Speranza (critica y narradora)

Alberto Goldenstein (fotógrafo)

Jorge Macchi (artista visual)

Mariana Obersztern (dramaturga)

Alejandro Ariel (psicoanalisma)

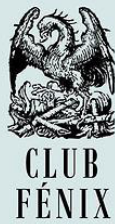

Convocatoria

El trabajo en Club Fénix está dirigido a artistas de todas las áreas: artistas visuales, escultorss, pintorxs, fotógrafxs, escritorxs, performers, bailarinxs, poetas, dramaturgxs, cineastas, músicss, etc. Para ser unx fracasante de esta primera edición de Club Fénix, en el ciclo "Terror en verano", es necesario presentar un portfolio que incluya:

- Datos personales: Además de los usuales, nos encontramos en condiciones de aceptar otra serie de datos personales que releven aspectos que consideren relevantes: signo del zodiaco si han desarrollado pensamiento mágico, descripciones de su entorno de trabajo si son infuen (o - Antimanifiesto: Una presentación que puede ser textual, visual, audiovisual, sonora, etc., que manifieste las debilidades, inconsistencias, falta de seguridad sobre temas, practicas, metodologias, etc.: un antimanifiesto no (se) defiende, no (se) vende; un antimanifiesto se entrega.

- Un problema vinculado a un proyecto, una obra, un aspecto, una metodologia, una fantasia artística o una idea general sobre su propio desempeño que deseen sea tratado por un descifrante. Club Fénix se ha propuesto concentrarse en los problemas, obstriculos, inconvenientes, contrariedades, atolladeros o bretes de la producción. Ese serí el núcleo del intercambio durante los encuentros, el sujeto de las oraciones.

Este material debe ser enviado al mail ohclubfenix@gmail.com hasta el 16 de enero de 2018. Serí evaluado por un jurado que seleccionara a 12 fracasantes cuyos problemas presenten una correspondencin porence lxs demás fracasantes.

Se evaluará la descripción de los problemas, su potencialidad para

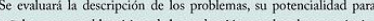
serial. mas. Los resultados de la convocatoria se comunicarán el día 22 de

Deseamos manifestar que no hay promesa ni perspectiva hacia el final de cada ciclo, sino la más sencilla consecución de la vida (en los términos en los que prefieran pensarla: naturalmente, históricamente. emocionalmente, colectivamente, individualmente, etc.). Es decir, no habrí muestra, libro, conclusiones, moralejas ni festejos; solo la continuidad de los dias de sus vidas en su aspecto menos heroico.

Contacto:

Contacto:
Serán bien recibidos comentarios $y$ consultas escuetas, sobrias $y$

directas en nuestro mail: ohclubfenix@gmail.com

(0) $\mathbf{t}$ 


\section{EL FRACASO COMO SABER VÁLIDO EN EL PROYECTO CLUB FÉNIX ${ }^{1}$}

"Club Fénix trata de replegar esfuerzos hacia zonas oscuras y negativizadas de la producción con el objetivo de visibilizar aquellas zonas áridas, secas o empantanadas, en un entorno respetuoso de trabajo, de baja estridencia y con concentración. Dos participantes o fracasantes expondrán un problema en una obra, un proyecto (o un aspecto) que no funcione, que se encuentre paralizado, inconsistente, débil en su concepción y/o realización y/o consecución (...) Recibirán, por parte de un descifrante una mirada, un indicio hacia una zona de excavación, un martilleo serio en los espacios fosilizados, la pista hacia un emplazamiento en el que hundirse para poder explorar las paredes que lo sostienen".

Dirigido y diseñado por dos artistas, Ayelen Coccoz y Mariana Lerner, la propuesta de Club Fénix tiene como objetivo principal dar espacio a la discusión acerca de los problemas que atraviesa la creación artística a través de una muestra performativa de lo que ocurre y no se muestra dentro del quehacer artístico. Renuncian a orientar el encuentro a la elaboración de un statement, exposición o catálogo, ya que consideran que esto desviaría el objetivo principal de su proyecto: aprender a valorar el fracaso, los obstáculos y dificultades inherentes al proceso creativo.

El proyecto Club Fénix es un espacio experimental en el que sus participantes comparten los problemas y las dudas que supone la práctica creativa. Exponiendo sus vicisitudes dan la oportunidad a que descifrantes y expectantes les ofrezcan sus observaciones y comentarios sobre "las áreas más bloqueadas, amenazadas, destructivas y paralizantes de la propia producción".El fracasante, artista participante, comienza su aportación con la elaboración de un antimanifiesto, es decir piensa y diseña, la manera de mostrar y poder compartir el mapa del no, lo que no ha funcionado en su quehacer artístico.

Organizado en seis encuentros por ciclo que tienen lugar cada quince días, se desarrolla en Buenos Aires, Argentina, a lo largo de cuatro ciclos diferentes: Terror en verano, Pavor en Otoño, Temor en invierno y Pánico en primavera. En los enunciados de cada ciclo se dejan ver ya los intereses y signos de la deriva del Club Fénix, humor y cierto desafío a los miedos que operan en la sensación de fracaso de todo proceso creativo. 
El grupo está compuesto por 12 fracasantes, 12 artistas de diferentes disciplinas, que presentan su problema o fracaso ante un descifrante. Figura, la del descifrante, desempeñada por artista o agente vinculado al arte, provenientes estos también de diversas disciplinas. El papel del descifrante es analizar y comentar sus impresiones sobre la exposición del caso presentado por el fracasante. Todo se desarrolla con cierto carácter performativo. Además de los fracasantes y el descifrante, asisten también los expectantesque aunque no pueden intervenir de manera oral, pueden hacerlo de forma escrita. Ese texto, que los expectantes producen como observación de la puesta en diálogo entre fracasante y descifrante, es luego compartido en un espacio virtual que circula entre los participantes del ciclo.

"Si bien C.F. se trata de un dispositivo de discusión sobre la práctica artística, el dispositivo en sí mismo es una obra, una especie de performance. Un espacio guionado por nosotras (a través de las muchas reglas, la vestimenta, las bebidas que ofrecemos, la escenografía del espacio que utilizamos, las coreografías que desplegamos, la forma en que nombramos los diferentes roles fracasantes, descifrantes, expectantes, durmientes-) pero donde cada encuentro es de alguna forma una experiencia única“.

En todo momento, y desde el lenguaje que construye y da significado al proyecto del Club Fénix, se apuesta por generar un espacio honesto y de inmersión en la problemática de la práctica artística. Se persigue habilitar un lugar, en el que la duda pueda tener presencia porque no hay pudor ni falta de rigor investigador en su manifestación, sino que la puesta en escena de esta permite precisamente articular ese espacio natural al proceso creativo en el que hay titubeos, merodeos y acercamientos infructuosos.

\section{CATÁBASIS. APROXIMACIÓN A LA EXTENSIÓN OCULTA DEL PROCESO CREATIVO}

Aunque reivindicamos la práctica artística como un ejercicio desde el que se puede y se debe investigar, los protocolos académicos, la certeza y la razón siguen siendo el lugar que perseguimos como investigadores y creadores de conocimiento. Sin embargo, lejos de esto, nuestra práctica artística y nuestras formas de hacer están más próximas a métodos que dinamitan las cer- 
tezas y el pensamiento lineal. "La investigación avanza sobre esa necesidad de comunicar lo incomunicable" señalaba Mika Elo durante su conferencia en las Jornadas sobre investigación artística basada en la práctica organizadas por LaSia en $2018^{2}$, señalando así una de las cuestiones paradójicas del arte. Este lugar cargado de contradicciones que ocupa la práctica artística es problematizado, dentro y fuera de la academia. Borgdoff (2006) se refería a "La dificultad a la que se enfrentan los candidatos de doctorado y sus supervisores para evitar el escepticismo sobre las investigaciones basadas en la práctica". Si bien el proceso artístico propone y dispone de otras herramientas metodológicas para desplegar un saber, es cierto que continuamente padece el cuestionamiento y/o negación por parte de sus "propios militantes" de la puesta en evidencia de estos "merodeos" que nos conducen en la práctica artística. Como si las dudas y titubeos del proceso investigador del artista se trataran de un defecto a salvar y no de una herramienta y material poderoso para repensar los mundos que nos habitan. Como si el despliegue de ese "conocimiento intuitivo" del que hablaba Maria Zambrano no se tratara de un lugar-umbral desde el que detenerse a ver el mundo.

La problematización comienza con nuestro propio concepto de la construcción de conocimiento y saber, y continua con nuestra concepción del arte y sus posibilidades metodológicas. Ya desde el siglo XIX queda fuera de la investigación el cuerpo, es decir, lo sensorial y lo sentido; la subjetividad; la ficción y la imaginación. Tomando en cuenta este contexto científico las preguntas que surgen vienen a confirmar la controversia que sufre la práctica artística y contra la que se debate, ¿qué tipo de saber es el arte? ¿cómo legitimamos ese saber, ese deseo de hacer arte, "una actividad que supone una radical implicación del sujeto en la observación" (Moraza 2009)? Un saber que es producido, planteado y desarrollado por el mismo sujeto, pone en solfa muchas de las consideraciones establecidas en el conocimiento académico: la no distancia entre el investigador y el objeto investigado, la pertinencia/repercusión o no del fruto de su investigación para la academia, el estilo como método y forma de conocimiento. El carácter reflexivo del arte, la necesidad de comunicabilidad de lo incomunicable y una metodología que no es discursiva hacen compleja la tarea. Dirigirnos, especialmente a un afuera donde el "hacer arte" no ha sido experienciado, y afirmar que nada tiene que ver con lo arbitrario, es a veces tan complejo como debatirlo entre nosotros. ¿Cómo salvamos la distancia entre códigos que no son compartidos?

El Fracasante en el momento de su intervención comparte un saber que podríamos ilustrar haciendo uso del término catábasis. Catábasis, un descenso 


\section{Producir}

\section{no es defender (se)}

no es vender (se)

es entregar (se)

es pensar (nos)

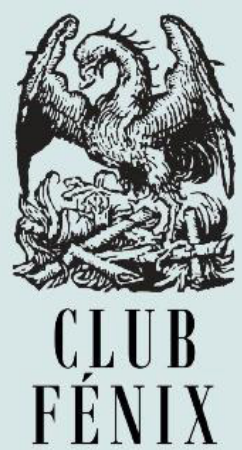


hacia las bajas pasiones, hacia el cuerpo. Una búsqueda que puede terminar en una anábasis, camino ascendente desde un abismo que hace comunicable el saber adquirido: Platón y su mito de la caverna, Virgilio y Dante, Eneas, etc. Es decir, se baja a lo profundo (catábasis) para volver a subir y comunicar lo descubierto (anábasis).

El fracaso es una pulsión que sobrevuela constantemente el proceso artístico. La consciencia de la inexactitud entre lo que uno quiere decir y lo que le llega al espectador es como la cuerda por la que camina el funambulista. La catábasis ilustra buena parte del saber que construimos desde el arte, un saber difícil de comunicar y sobre todo, opacado y difícil de reconocer si no culmina en anábasis, es decir, en el éxito del hacer. La elaboración de nuestro trabajo conoce múltiples formas metodológicas, pero podríamos afirmar que en términos generales se debate y tensiona constantemente entre: la acción que responde al propio proceso de trabajo y la imagen que habíamos proyectado como resolución. Podemos comenzar desde un punto azaroso, desde un caso de estudio, desde una cuestión formal. El titubeo, el merodeo, la necesidad de salvar el obstáculo de lo indecible, verdadero acicate de la práctica, obliga a trazar una estrategia. La consciencia de la imprecisión del mensaje guía la investigación, la práctica y la acción. llustrar lo obvio no nos sirve, tampoco limitarnos a relatar nuestro propósito. El "hacer" está determinado por la conversación que se tiene con materiales y/o campo de estudio y las resistencias que estos ofrecen. Estas resistencias, resultan de alguna manera estimulantes y parte de un filtro de exigencias sobre las que se despliega el trabajo.

Sin embargo, estas maneras/experiencias de descenso, catábasis, se ven normalmente opacadas sino censuradas por la articulación de un discurso coherente y lineal, heredero de protocolos académicos. Las menciones "procesuales" son sólo recuperadas puntualmente si es para presentarnos los albores de un objeto, obra o proceso llegado a término, anábasis. El fomento de espacios que puedan ofrecer a los artistas y estudiantes de arte, de herramientas y espacios vinculados a la práctica, en los que esta se someta al escrutinio generoso de quien comparte sus miedos y sus fracasos, sería un primer paso hacia la puesta en valor de modos de conocimiento y metodologías a partir de la praxis artística. La dificultad que entraña encontrar un método de comunicabilidad, es el germen de la obra de arte, catalizador del proceso de investigación y también buena parte de nuestra producción de saber. Si no le reconocemos el lugar que ocupa caemos en el riesgo de que la obra termine como mera ilustración del proyecto del artista. 


\section{LA OBRA (ANÁBASIS) COMO TESTIGO PARCIAL DE UN SABER MÁS EXTENSO QUE QUEREMOS REVINDICAR}

La obra como tal es una reflexión del artista, sujeto investigador, es al mismo tiempo proceso y puesta en exposición de un proceso. El recorrido que atraviesa la obra en sí yque ha permitido llegar hasta ella raramente es lineal. Su producción es un proceso complejo que supone el desafío de dejarse llevar por "el hacer". Cada tanto uno se para a observar hasta donde le ha llevado ese "hacer", siendo esta la tensión principal en la que se enmarca el proceso artístico. Tensión entre lo que dejamos que suceda y la idea original o imagen que proyectamos como resultado final, origen que es un espacio acotado y a superar. Todas las decisiones, las renuncias, lo que uno quiere decir, lo que proyectaba, lo que negocia con la pieza cuando va ganando identidad propia etc, convergen en ella y sin embargo no podemos considerarla como prueba documental de la práctica o como prueba que ilustra esa investigación que nos ha permitido llegar hasta ella. La obra es un medio camino entre lo explorado y lo que nos conmueve, pero nuestra formación cultural entiende que la comunicación de la experiencia sólo cabe dentro del sentido en el que media el control, cuando en nuestra práctica artística el enriquecimiento es dado también por la falta de control, por la aparición súbita en el hacer de lo inesperado, de lo no proyectado. La obra es como un desprendimiento que dejamos para el espectador, reconociendo esto reconoceriamos que el proceso investigador, el camino que se recorrió y el que no se hizo, queda sólo para el artista. ¿Cuáles serían las consecuencias de dar a conocer ese espacio de catábasis?

Las reformas educativas como el plan Bolonia, en las que la investigación ha tomado un lugar central, se han preguntado con más apremio si la práctica artística es parte de una investigación cuyo resultado es la obra, y si esta investigación siendo diferente de otras puede y/o debe estar dentro del campo académico. En la performatividad de la propuesta de Club Fénix podríamos tener un ejemplo que opera para poner en valor algunas cuestiones aún por reivindicar. Nos permitirían desvelar partes de la praxis artística como construcción de saber que quedan ocultas, desconocidas. Además del conocimiento que se produciría a través de las obras mismas, estaríamos hablando también del saber que las contextualiza, no tanto por la mediación de lo explicativo de su producción sino por el recorrido de toma de decisiones en las que 
se inscriben, incluidas todas las que no resultaron, lo cual exige el reconocimiento de una manera de saber propiamente artística.

"El arte no solo se define por lo que es o no es, por lo que hace o puede hacer', escribió la directora artística de DOCUMENTA, Carolyn Christov-Bakargiev, 'sino también por lo que no hace o no puede hacer, e incluso por lo que quiso hacer y no pudo'"

[Graciela Speranza, primera descifrante, durante el ciclo Terror en Verano].

La investigación artística se presenta en público consciente de la incapacidad de registro total o veraz del proceso. Se trata de hacerla comunicable aun a sabiendas de que algunas de las dimensiones del proceso no se ajustan a dimensiones escritas u orales. Un arte que siempre es reflexivo, si además pretende ser investigador "tiene que estar dispuesto a compartir de manera clara y concreta lo que hemos trabajado", sugería Janneke Wesseling (LaSia $2018^{3}$ ), subrayando a continuación la importancia de la escritura como forma de investigación, la necesidad de que la escritura acompañe a la práctica y forme parte de ella. La escritura, explicaba, funciona como un lugar desde el que no evitamos lo subjetivo que pueda tener la investigación, pero concedemos al sujeto una vía desde la que tomar distancia para repensar el hacer. A la cuestión de la escritura y su pertinencia en el proceso investigador en el caso del Club Fénix se procede de la siguiente manera:

"La idea de que los expectantes no hablen, pero sí pueden escribir, tiene varios aspectos. Al principio nos gustaba la idea de que los cobardes (los que no se animaban a mostrar su fracaso) pudieran participar de algún modo. Después se nos ocurrió que la figura del expectante podría ser un buen lugar para quienes se acercan a la práctica artística de forma más teórica, pero sobre todo, para dar lugar a los posibles registros de la experiencia subjetiva de cada encuentro, que no se graban ni se registran de modo alguno. Ahí los expectantes cobran también un rol protagónico, porque los registros que quedan son sus escritos. Los escritos se suben a una plataforma a la cual tienen acceso los participantes de C.F."

La obra tiene como interfaz una llegada para quien la contempla, que no tiene el texto. Aunque el título de las obras cobra a veces un papel mediador entre artista y espectador y puede presentarse como quien tira un mensaje embotellado a través de la cartela, apenas es un atisbo de todo lo que sostiene. 
La obra apoyada en el límite de las posibilidades, es terminada por lo que uno pone en ella como espectador, quedando abierta a lecturas e interpretaciones desde diversos ámbitos. Un artefacto que alude y sugiere porque pone en conexión, por relación simbólica, elementos que previamente se les confería un significado diferente. "La ventaja del arte es que muestra las cosas, no habla de ellas. Bueno también habla pero fundamentalmente nos pone delante algo" (Badiola 2017).Sin embargo, en este artículo pretendemos señalar que el saber se despliega no sólo en la obra, sino también en los fracasos y merodeos que previamente nos han acercado-alejado hasta llegar a ella y por lo tanto podríamos decir en este sentido que la obras se asemeja a un testigo de algo más amplio que queremos reivindicar como parte de un saber.

\section{EPÍLOGO}

"No creemos en muchas cosas, pero tenemos la sospecha de que el abandono del anichamiento de la mirada ayudará a dejar al descubierto (a la intemperie, al desamparo) los temas de la obra. Para eso, y porque entendemos que a la vez que se reproducen los discursos sobre las obras, hay una imposibilidad fundante de comunicar aquello de lo que la obra trata, hemos convocado a personas de las más diversas áreas para que miren, miren, miren, piensen, piensen, piensen y hablen, hablen, hablen. Una devolución siempre en negativo -así el espíritu de Club Fénix-, una devoIución centrada en indagar aquello que efectivamente no funciona, lo que queda fuera y debería entrar, lo que entra y debería salir, en definitiva, un mapa de un problema, un mapa del no".

La propuesta del Club Fénix, diseñando estrategias de investigación que centran la mirada en aquello que queda a la sombra de la producción, expande nuestra formulación y metodologías del saber, dándonos la oportunidad de acceder a nuevos modos de conocimiento a partir de la praxis artística.

Club Fénix construye conocimiento desde la validación del fracaso como herramienta para construir un saber. Una puesta en valor del quehacer artístico que incluye el fracaso, la búsqueda, los proyectos inconclusos, catábasis, permite generar una experiencia que amplía el marco de construcción de saber que genera la práctica artística. Tomamos este ejemplo de investigación 
interdisciplinar como fórmula para promover nuevos modos de conocimiento desde nuestra práctica y como estrategia para repensar las posibilidades de su comunicación. Creemos que desde el ejercicio performativo y la puesta en común para compartir la catábasis, (la parte oculta, la parte oscura, lo que está a la sombra de la producción, lo que no se muestra) se ponen en evidencia los problemas que atraviesa la creación artística, la inercia que determina nuestro concepto de aprendizaje y saber, y la necesidad de buscar nuevos modelos para su comunicación. Entendemos que fomentar nuevas formas de exponer nuestro proceder es necesario no sólo por lo que diferencia la investigación artística de la investigación académica predominante sino por lo que esto enriquecería nuestra sociedad y nuestro conocimiento. En la actualidad la presentación pública de la práctica artística como investigación, se da desde protocolos heredados y pautas establecidas, muestras de seguridad, convicción y obra. Faltan espacios en los que poder compartir con otros artistas nuestras derivas procesuales, dudas y frustración. La práctica artística, en tanto proceso que construye saber, busca legitimarse desde otros modelos comunicativos, lo cual requiere que seguir avanzando en el estudio de estos conceptos de fracaso y catábasis en cuanto a su significación.

El reto ahora consiste en articular dentro de los protocolos académicos este espacio a la duda, abrazar esta cuestión paradójica desde estos mismos espacios. Reconocer las calidades que nos ofrece un marco que no es estable, un tema que resulta escurridizo. Entendiendo que el saber que genera la práctica artística no es acumulativo sino relacional, y eso sitúa al artista investigador como aquel que concluye iniciando nuevas líneas de pensamiento y entrecruzamientos, que no da respuestas sino que formula nuevas preguntas.

\section{Referencias}

Borgdorff, Henk. (2005) 2010. "El debate sobre la investigación en las artes”. Cairon 13: 25-46

Badiola, Txomin.2017.“Arte, educación y amor al arte”. Eremuak4:15-24

Corazón Climent, Alberto. 2008. Una mirada en palabras. Madrid: Seix Barral

Groys, Boris. 2014. Volverse público: Las transformaciones del arte en el ágora contemporanea. Traducido por Matías Battistón. Buenos Aires: Caja Negra

Rogff, Irit. 2017.“Los desencantados”.Eremuak4: 45-9

Moraza Pérez, Jose Luis. (2009) 2011. El deseo del artista. Transcripción de Carmen Gallano.

En El deseo: Textos y conferencias. Madrid: Colegio de Psicoanálisis del Campo Lacaniano. www.victordelrio.es/blog_docente/wp-content/uploads/2013/04/EL-DESEO-DEL-ARTISTA.pdf

Sánchez Martínez, José Antonio. 2013. "In-definiciones: El campo abierto de la investigación en artes". Artes 19: 36-51 
Wesseling, Janneke. 2011. See it again, say it again: The artist as researcher. Amsterdam: Valiz. https://sabrinasoyer.files.wordpress.com/2016/05/janneke-wesseling-see-it-againsay-it-again-the-artist-as-researcher.pdf

Zweig, Stefan. (1953) 2012. El misterio de la creación artística. Traducido por Tristán La Rosa. Madrid: Sequitur

Notas

1 Club Fénix- páginas de información y acceso a materiales del proyecto: https://clubfenix.tumblr.com/, https://www.facebook.com/ohclubfenix/,https://www.instagram.com/ohclubfenix/

2 Mika Elo (University of the Arts Helsinki). Conferencia presentada en las Jornadas LaSia, sobre investigación artística basada en la práctica, 11 y 12 de enero de 2018 en la Sala Rekalde (Bilbao). Transcripción de la autora,

3 JannekeWesseling (Academy of Creative and Performing Arts, Leiden). Conferencia presentada en las Jornadas LaSia, sobre investigación artística basada en la práctica, 11 y 12 de enero de 2018 en la Sala Rekalde (Bilbao). Transcripción de la autora. 